\title{
A NOTE ON THE QUATERNION GROUP AS GALOIS GROUP
}

\author{
ROGER WARE
}

(Communicated by Louis J. Ratliff, Jr.)

\begin{abstract}
The occurrence of the quaternion group as a Galois group over certain fields is investigated. A theorem of Witt on quaternionic Galois extensions plays a key role.
\end{abstract}

In $[9, \S 6]$ Witt proved a theorem characterizing quaternionic Galois extensions. Namely, he showed that if $F$ is a field of characteristic not 2 then an extension $L=F(\sqrt{a}, \sqrt{b}), a, b \in F$, of degree 4 over $F$ can be embedded in a Galois extension $K$ of $F$ with $\operatorname{Gal}(K / F) \cong H_{8}$ (the quaternion group of order 8 ) if and only if the quadratic form $a x^{2}+b y^{2}+a b z^{2}$ is isomorphic to $x^{2}+y^{2}+z^{2}$. In addition he showed how to explicitly construct the Galois extension from the isometry. An immediate and interesting consequence of this is the fact that $H_{8}$ cannot be a Galois group over any Pythagorean field.

In this note Witt's theorem is used to obtain additional results about the existence of $H_{8}$ as a Galois group over certain fields. If $F$ is a field (of characteristic not 2) with at most one (total) ordering such that $H_{8}$ does not occur as a Galois group over $F$ then the structure of the pro-2-Galois groups $G_{F}(2)=\operatorname{Gal}(F(2) / F), G_{\mathrm{py}}=\operatorname{Gal}\left(F_{\mathrm{py}} / F\right)$ (where $F(2)$ and $F_{\mathrm{py}}$ are the quadratic and pythagorean closures of $F$ ) are completely determined. Moreover it is shown that for any field $F$ of characteristic not two, $H_{8}$ occurs as a Galois group over $F$ iff $H_{8}$ is a homomorphic image of $G_{\mathrm{py}}$ iff the dihedral group $D_{8}$ of order 8 is a homomorphic image of $G_{\mathrm{py}}$.

In what follows all fields have characteristic different from 2 . If $a_{1}, \ldots, a_{n} \in$ $\dot{F}=F \backslash\{0\}$ then $q=\left\langle a_{1}, \ldots, a_{n}\right\rangle$ denotes the quadratic form with orthogonal basis $e_{1}, \ldots, e_{n}$ and $q\left(e_{i}\right)=a_{i}$. The value set of $q$ is $D(q)=\{a \in \dot{F} \mid q(x)=a$ for some $x\}$. Any unexplained notations and terminology about quadratic forms can be found in [4].

Lemma 1. For a field $F$ with $-1 \notin F^{2}$ and $\left|\dot{F} / \dot{F}^{2}\right|>2$ the following are equivalent:

(1) The level (stufe), $s(F)$, of $F$ is two.

Received by the editors March 24, 1989.

1980 Mathematics Subject Classification (1985 Revision). Primary 11E81, 12F10; Secondary, $12 \mathrm{D} 15$.

Supported in part by NSA research grant No. MDA 904-88-H-2018. 
(2) Every quadratic extension of $F$ can be embedded in a quaternionic $G a-$ lois extension.

(3) $F(\sqrt{-1})$ is contained in a quaternionic Galois extension.

Proof. (1) $\Rightarrow(2)$. Let $a \in F, a \notin F^{2} \cup-F^{2}$. By $(1),\langle 1,1,1\rangle \cong\langle-1$, $-1,1\rangle \cong\langle-1, a,-a\rangle$. Hence by Witt's theorem $[9, \S 6], F(\sqrt{-1}, \sqrt{a})$ is contained in a quaternionic extension.

$(3) \rightarrow(1)$. By Witt's theorem, there exists $a \in \dot{F}$ such that $\langle 1,1,1\rangle \cong$ $\langle-1, a,-a\rangle$. Hence $\langle 1,1,1\rangle$ is isotropic and $s(F)=2\left(\right.$ as $\left.-1 \notin F^{2}\right)$.

An element $a$ in $\dot{F}$ is rigid if $a \notin \dot{F}^{2} \cup-\dot{F}^{2}$ and $D(\langle 1, a\rangle)=\dot{F}^{2} \cup a \dot{F}^{2}$.

Lemma 2. For $a \in D(\langle 1,1\rangle), a \notin F^{2}$, the following are equivalent:

(1) $a$ is not rigid

(2) $F(\sqrt{a})$ can be embedded in a quaternionic Galois extension.

Proof. $(1) \Rightarrow(2)$. As $a$ is not rigid, there exists $b \notin F^{2} \cup a F^{2}$ such that $\langle 1, a\rangle \cong\langle b, a b\rangle$. Hence $\langle 1,1,1\rangle \cong\langle 1, a, a\rangle \cong\langle b, a b, a\rangle$ and $[9, \S 6]$ applies.

$(2) \Rightarrow(1)$. By $[9, \S 6]$ there exists $b \in F \backslash\left(F^{2} \cup a F^{2}\right)$ such that $\langle a, b, a b\rangle \cong$ $\langle 1,1,1\rangle \cong\langle a, a, 1\rangle$ and by Witt's cancellation $\langle b, a b\rangle \cong\langle 1, a\rangle$. Hence $a$ is not rigid.

Remark. There exist fields with $s(F)=2$ such that all elements not in $F^{2} \cup-F^{2}$ are rigid (e.g. $\left.F=\mathbf{F}_{3}\left(\left(t_{1}\right)\right) \ldots\left(\left(t_{n}\right)\right)\right)$. Of course, for such fields $D(\langle 1,1\rangle)=$ $\dot{F}^{2} \cup-\dot{F}^{2}$ [7, Corollary 1.2].

Let $W F$ denote the Witt ring of anisotropic quadratic forms over $F$ and let $G_{F}(2)=\operatorname{Gal}(F(2) / F)$, where $F(2)$ is the maximal 2-extension of $F$. The next theorem improves Theorem 3.5 in [7]:

Theorem 1. For a field with $\left|\dot{F} / \dot{F}^{2}\right|>2$ the following are equivalent:

(1) $W F \cong \mathbf{Z} / 2 \mathbf{Z}\left[\dot{F} / \dot{F}^{2}\right]$

(2) $G_{F}(2)$ has (topological) generators $\left\{y_{i}, x\right\}_{i \in I}$ with relations $y_{i} y_{j}=y_{j} y_{i}$ and either $x y_{i} x^{-1}=y_{i}^{5^{m}}$ for fixed $m=2^{n}(n \geq 0)$ and all $i \in I$ or $x y_{i}=y_{i} x$ for all $i$. $F$.

(3) The dihedral group $D_{8}$ of order 8 does not occur as a Galois group over

(4) $F$ is not formally real and the quaternion group $H_{8}$ does not occur as a Galois group over $F$.

Proof. The equivalence of (1) and (3) as well as the implication (3) $\Rightarrow(4)$ is contained in [7, Th. 3.5].

$(1) \Rightarrow(2)$. If all 2-power roots of unity lie in $F$ then by [7, Cor. 3.9(2)] $G_{F}(2)$ has generators and relations as described with $x y_{i}=y_{i} x$ for all $i \in I$. Now assume $F$ does not contain all 2-power roots of unity. By [3, Ths. 2.1, 2.3, 
and Lemma 4.1(i)], $G_{F}(2)$ has the described generators and relations where $n \geq$ 0 is the largest integer such that $F$ contains a primitive $2^{n+2}$ th root of unity.

(2) $\Rightarrow(3)$. As $D_{8}$ is a 2-group, $D_{8}$ occurs as a Galois group over $F$ iff $D_{8}$ is a homomorphic image of $G_{F}(2)$. However, a pro-2-group with generators and relations described in (2) cannot have $D_{8}$ as a homomorphic image.

(4) $\Rightarrow(1)$. Assume (4). From Lemma 2 it follows that any sum of two squares in $F \backslash\left(F^{2} \cup-F^{2}\right)$ is rigid and hence a sum of three squares in $F$ can be written as the sum of two squares. Inductively it follows that $\dot{F}=D(\langle 1,1\rangle)$. Hence by Lemma 2 all elements in $F \backslash\left(F^{2} \cup-F^{2}\right)$ are rigid and by Lemma 1, $-1 \in F^{2}$. Statement (1) now follows from [7, Th. 1.5].

Corollary. Assume $F$ is not formally real. Then $D_{8}$ occurs as a Galois group over $F$ if and only if $H_{8}$ occurs over $F$.

Remark. If $G$ is a pro-2-group with generators and relations as described in Theorem 1 (2) there is a field $F$ with $G_{F}(2) \cong G$. This can be seen as follows:

If $G$ is not abelian let $\Gamma=Z^{(I)}$ (direct sum), let $K$ be a 2-extension of $\mathbf{Q}\left(e_{n+2}\right)$ maximal with respect to the exclusion of $e_{n+3}$, where $e_{k}$ is a primitive $2^{k}$ th root of unity, and let $F=K((\Gamma))$ be the generalized henselian power series field. If $G$ is abelian (with basis $\left\{y_{i}\right\}_{i \in I}$ ) take $F=\mathbf{C}((\Gamma))$. Then (in either case) $G_{F}(2) \cong G$ by [3, Th. 2.4].

Now let $F$ be formally real, let $F_{\text {py }}$ denote the pythagorean closure of $F$, and let $G_{\mathrm{py}}=\mathrm{Gal}\left(F_{\mathrm{py}} / F\right)$ denote the corresponding pro-2-Galois group. In [5], Minač showed that if $D_{8}$ is not a homomorphic image of $G_{\mathrm{py}}$ then neither is $H_{8}$. His argument used an equivalent form of Witt's theorem [2, 7.7 (ii)] (compare [6, Example, 663-664]) and improved Theorem 3.9 in [8] (answering a question raised in [8]). It should be pointed out that there is an oversight in the statement of [8, Theorem 3.9]; namely, the statement should include the assumption that $F$ is formally real (the observation on lines 2-3 of page 104 of [8] is false if $F$ is nonreal of level 2). The next theorem improves Minač's theorem.

Theorem 2 (cf. [5, Th. 2], [8, Th. 3.9]). For a formally real field the following are equivalent:

(1) If $t \in F \backslash F^{2}$ is a sum of squares then $t$ is rigid.

(2) $D_{8}$ is not a homomorphic image of $G_{\mathrm{py}}$.

(3) $H_{8}$ does not occur as a Galois group over $F$.

(4) $H_{8}$ is not a homomorphic image of $G_{\mathrm{py}}$.

Proof The equivalence of (1) and (2) is contained in [8, Th. 3.9] while the equivalence of (1) and (3) follows from Lemma 2. It remains to prove (4) $\Rightarrow(3)$ :

Assume there exists a Galois extension $K / F$ such that $\operatorname{Gal}(K / F) \cong H_{8}$. Then there exist $a, b$ in $F$, independent mod squares, such that $F(\sqrt{a}, \sqrt{b}) \subseteq$ $K$. By $[9, \S 6]\langle a, b, a b\rangle \cong\langle 1,1,1\rangle$. Hence $F(\sqrt{a}, \sqrt{b}) \subseteq F_{\text {py }}$ so there is an 
epimorphism $f: G_{\mathrm{py}} \rightarrow V=\operatorname{Gal}(F(\sqrt{a}, \sqrt{b}) / F)$ and a diagram

$$
1 \rightarrow \mathbf{Z} / 2 \mathbf{Z} \rightarrow H_{8} \stackrel{h}{\rightarrow} V \stackrel{\rfloor_{\mathrm{py}}}{\rightarrow}
$$

with exact row. Let $e \in H^{2}(V, \mathbf{Z} / 2 \mathbf{Z})$ correspond to the above row. It is well known that there is a surjective homomorphism $\bar{f}: G_{\mathrm{py}} \rightarrow H_{8}$ such that $h \circ \bar{f}=f$ if and only if $f^{*}(e)=0$ where $f^{*}: H^{2}(V, \mathbf{Z} / 2 \mathbf{Z}) \rightarrow H^{2}\left(G_{\mathrm{py}}, \mathbf{Z} / 2 \mathbf{Z}\right)$ is induced by $f$ (cf., $[2, \S 7],[6, \S 3]$ ).

Let $G_{F}$ be the absolute Galois group of $F$, let $s: G_{F} \rightarrow G_{\text {py }}$ be the natural surjection, and let $g=f \circ s$. Then if $\bar{g}: G_{F} \rightarrow \operatorname{Gal}(K / F) \cong H_{8}$ is the natural map, we have $g=h \circ \bar{g}$. Hence $g^{*}(e)=0$ in $H^{2}\left(G_{F}, \mathbf{Z} / 2 \mathbf{Z}\right)$. By [8, Cor. 2.2], $H^{2}\left(G_{\mathrm{py}}, \mathbf{Z} / 2 \mathbf{Z}\right) \rightarrow B r\left(F_{\mathrm{py}} / F\right) \subseteq B r(F)$ is injective, whence $s^{*}: H^{2}\left(G_{\mathrm{py}}, \mathbf{Z} / 2 \mathbf{Z}\right) \rightarrow H^{2}\left(G_{F}, \mathbf{Z} / 2 \mathbf{Z}\right) \cong B r_{2}(F)$ is injective. As $g^{*}=s^{*} \circ f^{*}$ we conclude that $f^{*}(e)=0$. Hence $H_{8}$ is a homomorphic image of $G_{\mathrm{py}}$, completing the proof of Theorem 2.

An extension $K / F$ is called totally positive if every ordering (if any) on $F$ extends to an ordering on $K$.

Corollary. For a field $F$ the following are equivalent:

(1) $H_{8}$ occurs as a Galois group over $F$.

(2) There is a totally positive Galois extension $K / F$ such that $\mathrm{Gal}(K / F) \cong$ $\mathrm{H}_{8}$.

(3) There is a totally positive Galois extension $L / F$ such that $\mathrm{Gal}(L / F) \cong$ $D_{8}$.

Proof. It is well known that a 2-extension $K / F$ is totally positive iff $K \subseteq F_{\mathrm{py}}$.

Theorem 3. For a uniquely ordered field $F$ with positive cone $P$ the following are equivalent:

(1) $W F \cong \mathbf{Z} \times \mathbf{Z} / 2 \mathbf{Z}\left[P / \dot{F}^{2}\right]$, the fibre product over $\mathbf{Z} / 2 \mathbf{Z}$ (= product in the category of Witt rings).

(2) $G_{F}(2) \cong \mathbf{Z} / 2 \mathbf{Z} * G_{\mathrm{py}}$ (free pro-2-product) and $G_{\mathrm{py}}$ has (topological) generators $\left\{y_{i}, x\right\}_{i \in I}$ with relations $y_{i} y_{j}=y_{j} y_{i}$ and either $x y_{i} x^{-1}=y_{i}^{5^{m}}$ for fixed $m=2^{n} \quad(n \geq 0)$ and for all $i \in I$ or $x y_{i}=y_{i} x$ for all $i$.

(3) $G_{\mathrm{py}}$ has generators and relations as described in (2).

(4) $H_{8}$ does not occur as a Galois group over $F$.

Proof. (1) $\Rightarrow(2)$. By [1], Realization Theorem 4.8 and Remarks 4.9(i) there exist 2-extensions $K, L$ of $F$ such that $W K \cong \mathbf{Z}, W L \cong \mathbf{Z} / 2 \mathbf{Z}\left[P / \dot{F}^{2}\right]$, 
and the inclusions $F \subseteq K, L$ induce the isomorphisms $W F \cong W K \times W L \cong$ $\mathbf{Z} \times \mathbf{Z} / 2 \mathbf{Z}\left[P / \dot{F}^{2}\right]$. By [3, Th. 3.4], $G_{F}(2) \cong G_{K}(2) * G_{L}(2) \cong \mathbf{Z} / 2 \mathbf{Z} * G_{L}(2)$ and by Theorem $1, G_{L}(2)$ has the generators and relations described in (2).

Let $I_{t} F$ denote the torsion subgroup of the fundamental ideal $I F$ of $W F$. As $W F \cong \mathbf{Z} \times W L$ the inclusion $F \subseteq L$ induces an isomorphism $I_{t} F \rightarrow I L=$ $I_{t} L$ whence by $\left[8\right.$, Th. 2.10], $G_{\mathrm{py}} \cong G_{L}(2)$.

(3) $\Rightarrow(4)$. A pro-2-group with generators and relations as described in (2) cannot have $H_{8}$ as a homomorphic image. By Theorem 2, $H_{8}$ does not occur as a Galois group over $F$.

$(4) \Rightarrow(1)$. As $F$ is uniquely ordered, $P$ is the set of nonzero sums of squares and $(\dot{F}: P)=2$. Hence the mapping $\mathbf{Z}\left[P / \dot{F}^{2}\right] \rightarrow W F$ via $\sum n_{i}\left[t_{i}\right] \rightarrow$ $\sum n_{i}\left\langle t_{i}\right\rangle$ is surjective and by Theorem $2(1)$, its kernel is additively generated by the elements $2[t]-2[u], t, u \in P$. On the other hand, $\mathbf{Z}\left[P / \dot{F}^{2}\right] \rightarrow \mathbf{Z} \times$ $\mathbf{Z} / 2 \mathbf{Z}\left[P / \dot{F}^{2}\right]$ via $\sum n_{i}\left[t_{i}\right] \rightarrow\left(\sum n_{i}, \sum \bar{n}_{i}\left[t_{i}\right]\right)$ is surjective and $\sum n_{i}\left[t_{i}\right]$ lies in the kernel iff all $n_{i}$ are even and $\sum n_{i}=0$. This happens iff $\sum n_{i}\left[t_{i}\right]=$ $\sum 2\left(\left[u_{j}\right]-\left[v_{j}\right]\right)$, proving $(1)$.

Remark. If $G$ is a pro-2-group with generators and relations described in Theorem 3 (2) then by the remark following Theorem 1 and [8, Th. 4.1] there is a uniquely ordered field $F$ with $G_{\text {py }} \cong G$.

\section{REFERENCES}

1. J. Arason, R. Elman and B. Jacob, Rigid elements, valuations and realizations of Witt rings, J. Algebra 110 (1987), 449-467.

2. A. Fröhlich, Orthogonal representation of Galois groups, Stiefel-Whitney classes and Hasse-Witt invariants, J. Reine Angew. Math. 360 (1985), 84-123.

3. B. Jacob and R. Ware, A recursive description of the maximal pro-2-Galois group via Witt rings, Math. Z. 200 (1989), 379-396.

4. T. Y. Lam, The algebraic theory of quadratic forms, Benjamin, Elmsford, New York, 1973.

5. J. Minač, Quaternion fields inside the Pythagorean closure, J. Pure Appl. Algebra 57 (1989), 79-82.

6. J.-P. Serre, L'invariant de Witt de la Forme $\operatorname{Tr}\left(x^{2}\right)$, Comment Math. Helv. 59 (1984), 651676.

7. R. Ware, When are Witt rings group rings? II, Pacific J. Math. 76 (1978), 541-564.

8. - Quadratic forms and pro- 2-groups II: the Galois group of the Pythagorean closure of a formally real field, J. Pure Appl. Algebra 30 (1983), 95-107.

9. E. Witt, Konstruktion von galoisschen Körpen der Charakteristik $p$ zu vorgegebener Gruppe der Ordnung $p^{f}$, J. Reine Angew. Math. 174 (1936), 237-245.

Department of Mathematics, The Pennsylvania State University, University Park, PenNSylVANia 16802 